\title{
Effective gluon potential and Yang-Mills thermodynamics
}

\author{
Chihiro Sasaki* \\ Frankfurt Institute for Advanced Studies, D-60438 Frankfurt am Main, Germany \\ E-mail: sasaki@fias.uni-frankfurt.de
}

\section{Krzysztof Redlich}

Institute of Theoretical Physics, University of Wroclaw, PL-50204 Wroctaw, Poland

E-mail: krzysztof.redlicheift.uni.wroc.pl

\begin{abstract}
We derive the Polyakov-loop thermodynamic potential in the perturbative approach to pure SU(3) Yang-Mills theory. The potential expressed in terms of the Polyakov loop in the fundamental representation corresponds to that of the strong-coupling expansion, of which the relevant coefficients of the gluon energy distribution are specified by characters of the SU(3) group. At high temperature, the potential exhibits the correct asymptotic behavior, whereas at low temperature, it disfavors gluons as appropriate dynamical degrees of freedom. To quantify the Yang-Mills thermodynamics in confined phase, we introduce a hybrid approach which matches the effective gluon potential to that of glueballs, constrained by the QCD trace anomaly in terms of dilaton fields.
\end{abstract}

Xth Quark Confinement and the Hadron Spectrum,

October 8-12, 2012

TUM Campus Garching, Munich, Germany

\footnotetext{
* Speaker.
} 


\section{Introduction}

The structure of the QCD phase diagram and thermodynamics at finite baryon density is of crucial importance in heavy-ion phenomenology. Due to the sign problem in lattice calculations, a major approach to a finite density QCD is based on effective Lagrangians possessing the same global symmetries as the underlying QCD. The $S U\left(N_{c}\right)$ Yang-Mills theory has a global $Z\left(N_{c}\right)$ symmetry which is dynamically broken at high temperature. This is characterized by the Polyakov loop that plays a role of an order parameter of the $Z\left(N_{c}\right)$ symmetry [1]. Effective models for the Polyakov loop were suggested as a macroscopic approach to the pure gauge theory $[2,3]$. Their thermodynamics is qualitatively in agreement with that obtained in lattice gauge theories [4]. Alternative approaches are based on the quasi-particle picture of thermal gluons [5]. When gluons propagating in a constant gluon background are considered, the quasi-particle models naturally merge with the Polyakov loops, that appear in the partition function, as characters of the color gauge group $[6,7,8,9,10]$.

In this contribution we show, that the $S U(3)$ gluon thermodynamic potential can be derived directly from the Yang-Mills theory and is expressed in terms of the Polyakov loops in the fundamental representation. We summarize its properties and argue that at hight temperatures, it exhibits the correct asymptotic behavior, whereas at low temperatures, it disfavors gluons [11]. We therefore suggest a hybrid approach to Yang-Mills thermodynamics, which combines the effective gluon potential with glueballs implemented as dilaton fields.

\section{Thermodynamics of hot gluons}

We start from the partition function of the pure Yang-Mills theory

$$
Z=\int \mathscr{D} A_{\mu} \mathscr{D C} \mathscr{D} \bar{C} \exp \left[i \int d^{4} x \mathscr{L}_{\mathrm{YM}}\right]
$$

with gluon $A_{\mu}$ and ghost $C$ fields. Following $[3,12]$ we employ the background field method to evaluate the functional integral. The gluon field is decomposed into the background $\bar{A}_{\mu}$ and the quantum $\check{A}_{\mu}$ fields,

$$
A_{\mu}=\bar{A}_{\mu}+g \check{A}_{\mu} .
$$

The partition function is arranged as

$$
\ln Z=V \int \frac{d^{3} p}{(2 \pi)^{3}} \ln \operatorname{det}\left(1-\hat{L}_{A} e^{-|\vec{p}| / T}\right)+\ln M\left(\phi_{1}, \phi_{2}\right),
$$

where $\hat{L}_{A}$ is the Polyakov loop matrix in the adjoint representation and the two angular variables, $\phi_{1}$ and $\phi_{2}$, represent the rank of the $S U(3)$ group. The $M\left(\phi_{1}, \phi_{2}\right)$ is the Haar measure

$$
M\left(\phi_{1}, \phi_{2}\right)=\frac{8}{9 \pi^{2}} \sin ^{2}\left(\frac{\phi_{1}-\phi_{2}}{2}\right) \sin ^{2}\left(\frac{2 \phi_{1}+\phi_{2}}{2}\right) \sin ^{2}\left(\frac{\phi_{1}+2 \phi_{2}}{2}\right),
$$

for a fixed volume $V$, which is normalized such that

$$
\int_{0}^{2 \pi} \int_{0}^{2 \pi} d \phi_{1} d \phi_{2} M\left(\phi_{1}, \phi_{2}\right)=1
$$


The first term in Eq. (2.3) yields the gluon thermodynamic potential

$$
\Omega_{g}=2 T \int \frac{d^{3} p}{(2 \pi)^{3}} \operatorname{tr} \ln \left(1-\hat{L}_{A} e^{-E_{g} / T}\right)
$$

where $E_{g}=\sqrt{|\vec{p}|^{2}+M_{g}^{2}}$ is the quasi-gluon energy and the effective gluon mass $M_{g}$ is introduced from phenomenological reasons.

We define the gauge invariant quantities from the Polyakov loop matrix in the fundamental representation $\hat{L}_{F}$, as

$$
\Phi=\frac{1}{3} \operatorname{tr} \hat{L}_{F}, \quad \bar{\Phi}=\frac{1}{3} \operatorname{tr} \hat{L}_{F}^{\dagger} .
$$

Performing the trace over colors and expressing it in terms of $\Phi$ and its conjugate $\bar{\Phi}$, one arrives at

$$
\Omega_{g}=2 T \int \frac{d^{3} p}{(2 \pi)^{3}} \ln \left(1+\sum_{n=1}^{8} C_{n} e^{-n E_{g} / T}\right),
$$

with the coefficients $C_{n}$,

$$
\begin{aligned}
& C_{8}=1, \\
& C_{1}=C_{7}=1-9 \bar{\Phi} \Phi, \\
& C_{2}=C_{6}=1-27 \bar{\Phi} \Phi+27\left(\bar{\Phi}^{3}+\Phi^{3}\right), \\
& C_{3}=C_{5}=-2+27 \bar{\Phi} \Phi-81(\bar{\Phi} \Phi)^{2}, \\
& C_{4}=2\left[-1+9 \bar{\Phi} \Phi-27\left(\bar{\Phi}^{3}+\Phi^{3}\right)+81(\bar{\Phi} \Phi)^{2}\right] .
\end{aligned}
$$

Thus, the gluon energy distribution is identified solely by the characters of the fundamental and the conjugate representations of the $S U(3)$ gauge group.

We introduce an effective thermodynamic potential in the large volume limit from Eq. (2.3) as follows:

$$
\Omega=\Omega_{g}+\Omega_{\Phi}+c_{0},
$$

where $\Omega_{g}$ is given by Eq. (2.8) and the Haar measure part is found as

$$
\Omega_{\Phi}=-a_{0} T \ln \left[1-6 \bar{\Phi} \Phi+4\left(\Phi^{3}+\bar{\Phi}^{3}\right)-3(\bar{\Phi} \Phi)^{2}\right] .
$$

The potential (2.10) has, in general, three free parameters; $a_{0}, c_{0}$ and the gluon mass $M_{g}$. They can be chosen e.g. to reproduce the equation of state obtained in lattice gauge theories. It is straightforward to see, that the result of a non-interacting boson gas is recovered at asymptotically high temperature. Indeed, taking $\Phi, \bar{\Phi} \rightarrow 1$ one finds

$$
\Omega_{g}(\Phi=\bar{\Phi}=1)=16 T \int \frac{d^{3} p}{(2 \pi)^{3}} \ln \left(1-e^{-E_{g} / T}\right) .
$$

On the other hand, for a sufficiently large $M_{g} / T$, as expected near the phase transition, one can approximate the potential as

$$
\Omega_{g} \simeq \frac{T^{2} M_{g}^{2}}{\pi^{2}} \sum_{n=1}^{8} \frac{C_{n}}{n} K_{2}\left(n \beta M_{g}\right)
$$


with the Bessel function $K_{2}(x)$. In the quasi-particle approach, the above result can also be considered as a strong-coupling expansion, regarding the relation $M_{g}(T)=g(T) T$ with an effective gauge coupling $g(T)$.

The effective action to the next-to-leading order of the strong coupling expansion is obtained in terms of group characters as [10],

$$
S_{\text {eff }}^{(\mathrm{SC})}=\lambda_{10} S_{10}+\lambda_{20} S_{20}+\lambda_{11} S_{11}+\lambda_{21} S_{21}
$$

with products of characters $S_{p q}$, specified by two integers $p$ and $q$ counting the numbers of fundamental and conjugate representations, and couplings $\lambda_{p q}$ being real functions of temperature. Making the character expansion of Eq. (2.13), one readily finds the correspondence between $S_{p q}$ and $C_{n}$ as

$$
C_{1,7}=S_{10}, \quad C_{2,6}=S_{21}, \quad C_{3,5}=S_{11}, \quad C_{4}=S_{20} .
$$

On the other hand, taking the leading contribution, $\exp \left[-M_{g} / T\right]$ in the expansion, the "minimal model" is deduced with

$$
\Omega_{g} \simeq-\mathscr{F}\left(T, M_{g}\right) \bar{\Phi} \Phi,
$$

where the negative sign is required for a first-order transition [10]. The function $\mathscr{F}$ can be extracted from Eq. (2.10) and the resulting potential is of the form widely used in the PNJL model [13, 14, $15,16]$.

\section{A hybrid approach}

Although the potential (2.10) describes quite well thermodynamics in deconfined phase, it totally fails in the confined phase. In the confined phase, $\langle\Phi\rangle=0$ is dynamically favored by the ground state, thus the $C_{1}=1$ term remains as the main contribution. Consequently

$$
\Omega_{g}(\Phi=\bar{\Phi}=0) \simeq 2 T \int \frac{d^{3} p}{(2 \pi)^{3}} \ln \left(1+e^{-E_{g} / T}\right) .
$$

One clearly sees that $\Omega_{g}$ does not posses the correct sign in front of $\exp \left[-E_{g} / T\right]$, expected from the Bose-Einstein statistics. This implies that the entropy and the energy densities are negative. On the other hand, if one uses the approximated form (2.16), the pressure vanishes at any temperature below $T_{c}$. Obviously, this is an unphysical behavior since there exist color-singlet states, i.e. glueballs, contributing to thermodynamics and they must generate a non-vanishing pressure.

This aspect is in a striking contrast to the quark sector. The thermodynamic potential for quarks and anti-quarks with $N_{f}$ flavors is obtained as [13, 17]

$$
\Omega_{q+\bar{q}}=-2 N_{f} T \int \frac{d^{3} p}{(2 \pi)^{3}} \ln \left[1+N_{c}\left(\Phi+\bar{\Phi} e^{-E^{+} / T}\right) e^{-E^{+} / T}+e^{-3 E^{+} / T}\right]+(\mu \rightarrow-\mu),
$$

with $E^{ \pm}=E_{q} \mp \mu$ being the energy of a quark or anti-quark. In the limit, $\Phi, \bar{\Phi} \rightarrow 0$, the one- and two-quark states are suppressed and only the three-quark ("baryonic") states, $\sim \exp \left(-3 E^{( \pm)} / T\right)$, survives. This, on a qualitative level, is similar to confinement properties in QCD thermodynamics [15]. One should, however, keep in mind, that such quark models yield only colored quarks 
being statistically suppressed at low temperatures. On the other hand, unphysical thermodynamics below $T_{c}$ described by the gluon sector (2.10) apparently indicates, that gluons are physically forbidden. Interestingly, this property is not spoiled by the presence of quarks. Indeed, in this case and at $T<T_{c}$ the thermodynamic potential is approximated as

$$
\Omega_{g}+\Omega_{q+\bar{q}} \simeq \frac{T^{2}}{\pi^{2}}\left[M_{g}^{2} K_{2}\left(\frac{M_{g}}{T}\right)-\frac{2 N_{f}}{3} M_{q}^{2} K_{2}\left(\frac{3 M_{q}}{T}\right)\right] .
$$

Assuming that glueballs and nucleons are made from two weakly-interacting massive gluons and three massive quarks respectively and putting empirical numbers, $M_{\text {glueball }}=1.7 \mathrm{GeV}$ and $M_{\text {nucleon }}=$ $0.94 \mathrm{GeV}$, one finds that $M_{g}=0.85 \mathrm{GeV}$ and $M_{q}=0.31 \mathrm{GeV}$. Substituting these mass values in Eq. (3.3), one still gets the negative entropy density at any temperature and for either $N_{f}=2$ or $N_{f}=3$, as found in the pure Yang-Mills theory.

The unphysical equation of state (EoS) in confined phase can be avoided, when gluon degrees of freedom are replaced with glueballs. A glueball is introduced as a dilaton field $\chi$ representing the gluon composite $\left\langle A_{\mu v} A^{\mu v}\right\rangle$, which is responsible for the QCD trace anomaly [18]. The Lagrangian is of the standard form,

$$
\mathscr{L}_{\chi}=\frac{1}{2} \partial_{\mu} \chi \partial^{\mu} \chi-V_{\chi}, \quad V_{\chi}=\frac{B}{4}\left(\frac{\chi}{\chi_{0}}\right)^{4}\left[\ln \left(\frac{\chi}{\chi_{0}}\right)^{4}-1\right],
$$

with the bag constant $B$ and a dimensionful quantity $\chi_{0}$, to be fixed from the vacuum energy density and the glueball mass. One readily finds the thermodynamic potential of the glueballs as

$$
\begin{aligned}
\Omega & =\Omega_{\chi}+V_{\chi}+\frac{B}{4}, \quad \Omega_{\chi}=T \int \frac{d^{3} p}{(2 \pi)^{3}} \ln \left(1-e^{-E_{\chi} / T}\right), \\
E_{\chi} & =\sqrt{|\vec{p}|^{2}+M_{\chi}^{2}}, \quad M_{\chi}^{2}=\frac{\partial^{2} V_{\chi}}{\partial \chi^{2}},
\end{aligned}
$$

where a constant $B / 4$ is added so that $\Omega=0$ at zero temperature.

We propose the following hybrid approach which accounts for gluons and glueballs degrees of freedom by combining Eqs. (2.10) and (3.5),

$$
\Omega=\Theta\left(T_{c}-T\right) \Omega(\chi)+\Theta\left(T-T_{c}\right) \Omega(\Phi) .
$$

For a given $M_{g}$, the model parameters, $a_{0}$ and $c_{0}$, are fixed by requiring, that $\Omega(\Phi)$ yields a firstorder phase transition at $T_{c}=270 \mathrm{MeV}$ and that $\Omega(\chi)$ and $\Omega(\Phi)$ match at $T_{c}$. The resulting EoS follows general trends seen in lattice data [11]. The model can be improved further by introducing a thermal gluon mass, $M_{g}(T) \sim g(T) T$, as carried out e.g. in [8].

\section{Summary}

We have derived the thermodynamic potential in the $S U$ (3) Yang-Mills theory in the presence of a uniform gluon background field. The potential accounts for quantum statistics and reproduces an ideal gas limit at high temperature. Within the character expansion, the one-to-one correspondence to the effective action in the strong-coupling expansion is obtained. Different effective potentials used so far appear as limiting cases of our result. 
The phenomenological consequence is that gluons are disfavored as appropriate degrees of freedom in confined phase. This property is in remarkable contrast to the description of "confinement" within a class of chiral models with Polyakov loops $[13,16]$, where colored quarks are activated at any temperature. Further investigations of the $S U(3)$ gluodynamics guided by available lattice results with the effective gluon mass and a more realistic description of an effective QCD thermodynamics with quarks are desired.

\section{Acknowledgments}

C. S. acknowledges partial support by the Hessian LOEWE initiative through the Helmholtz International Center for FAIR (HIC for FAIR). K.R. acknowledges support by the Polish Science Foundation (NCN).

\section{References}

[1] L. D. McLerran and B. Svetitsky, Phys. Lett. B 98, 195 (1981); Phys. Rev. D 24, 450 (1981).

[2] R. D. Pisarski, Phys. Rev. D 62, 111501 (2000).

[3] R. D. Pisarski, hep-ph/0203271. A. Dumitru and R. D. Pisarski, Phys. Lett. B 525, 95 (2002). A. Dumitru, Y. Hatta, J. Lenaghan, K. Orginos and R. D. Pisarski, Phys. Rev. D 70, 034511 (2004)

[4] G. Boyd, J. Engels, F. Karsch, E. Laermann, C. Legeland, M. Lutgemeier and B. Petersson, Nucl. Phys. B 469, 419 (1996).

[5] A. Peshier, B. Kampfer, O. P. Pavlenko and G. Soff, Phys. Rev. D 54, 2399 (1996). M. Bluhm, B. Kampfer, R. Schulze and D. Seipt, Eur. Phys. J. C 49, 205 (2007). P. Levai and U. W. Heinz, Phys. Rev. C 57, 1879 (1998).

[6] L. Turko, Phys. Lett. B 104, 153 (1981). H. T. Elze, D. E. Miller and K. Redlich, Phys. Rev. D 35, 748 (1987). D. E. Miller and K. Redlich, Phys. Rev. D 37, 3716 (1988). K. Redlich, F. Karsch and A. Tounsi, hep-ph/0302245.

[7] P. N. Meisinger, T. R. Miller and M. C. Ogilvie, Phys. Rev. D 65, 034009 (2002), P. N. Meisinger and M. C. Ogilvie, Phys. Rev. D 65, 056013 (2002).

[8] P. N. Meisinger, M. C. Ogilvie and T. R. Miller, Phys. Lett. B 585, 149 (2004).

[9] K. Kusaka, Phys. Lett. B 269, 17 (1999).

[10] C. Wozar, T. Kaestner, A. Wipf, T. Heinzl and B. Pozsgay, Phys. Rev. D 74, 114501 (2006).

[11] C. Sasaki and K. Redlich, Phys. Rev. D 86, 014007 (2012) [arXiv:1204.4330 [hep-ph]].

[12] D. J. Gross, R. D. Pisarski and L. G. Yaffe, Rev. Mod. Phys. 53, 43 (1981).

[13] K. Fukushima, Phys. Lett. B 591, 277 (2004); Phys. Rev. D 68, 045004 (2003); Prog. Theor. Phys. Suppl. 153, 204 (2004). K. Fukushima and C. Sasaki, arXiv:1301.6377 [hep-ph].

[14] S. Roessner, C. Ratti and W. Weise, Phys. Rev. D 75, 034007 (2007).

[15] C. Sasaki, B. Friman and K. Redlich, Phys. Rev. D 75, 074013 (2007).

[16] C. Ratti, M. A. Thaler and W. Weise, Phys. Rev. D 73, 014019 (2006).

[17] E. Megias, E. Ruiz Arriola and L. L. Salcedo, Phys. Rev. D 74, 065005 (2006).

[18] J. Schechter, Phys. Rev. D 21, 3393 (1980). 